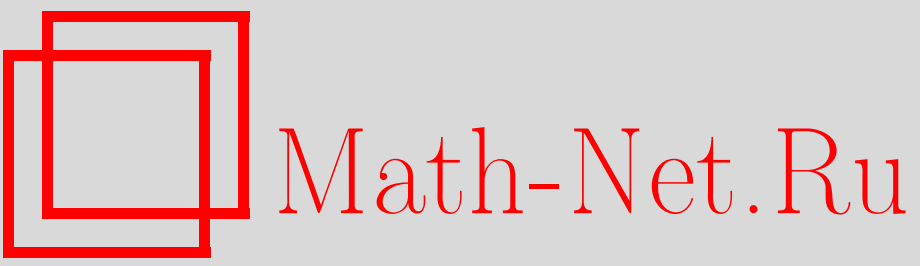

А. А. Андрианов, Д. Эсприу, Струны и пионы, ТМФ, 2003, том 135, номер 3, 355369

DOI: https://doi.org/10.4213/tmf194

Использование Общероссийского математического портала Math-Net.Ru подразумевает, что вы прочитали и согласны с пользовательским соглашением http://www.mathnet.ru/rus/agreement

Параметры загрузки:

IP: 52.6 .47 .48

26 апреля 2023 г., $11: 44: 22$ 
ТЕОРЕТИЧЕСКАЯ

И МАТЕМАТИЧЕСКАЯ

ФИЗИКА

Том 135, № 3

июнь, 2003

(C) 2003 г.

А. А. Андрианов ${ }^{*}$, Д. Эсприу ${ }^{\dagger}$

\section{СТРУНЫ И ПИОНЫ}

Предполагается, что КХД можно эффективно описать с помощью струноподобных переменных, и что в некоторой энергетической области такая струна приближенно представляется струной Намбу-Гото или Полякова. Однако для того, чтобы преодолеть давно существующие проблемы, связанные с поведением адронных струнных амплитуд при низких энергиях, струна строится надправильным (кирально-неинвариантным) КХД-вакуумом посредством граничного взаимодействия с фоновым киральным полем, связанным с пионами. Требуя совместности этого взаимодействия с конформной симметрией струны и с условиями унитарности для киральных полей, мы воспроизводим уравнения движения для последних и, более того, лагранжиан нелинейной сигма-модели пионных взаимодействий. В следующем за главным порядке в разложении по производным получены киральные структурные константы Гассера и Лейтвилера. Оценки для коэффициентов находятся в хорошем соответствии с феноменологическими значениями.

Ключевые слова: квантовая хромодинамика, пионные взаимодействия, адронные струны, конформная и киральная симметрии.

\section{1. ВВЕДЕНИЕ}

История попыток описания адронов в рамках струнной теориии вне пределов или в пределах КХД насчитьвает уже более 30 лет (см. [1]-[6], а также обзоры [7]-[10] и приведенный в них неполный список литературы). В настоящей работе мы концентрируем внимание исключительно на задаче адронизации КХД с использованием струнньх переменных.

Обычно для обоснования струнного описания КХД приводятся следующие аргументы: доминантность планарных глюонных диаграмм в пределе больших $N$ [3], "заполняющих" поверхность (интерпретируемую как мировой лист струн), разложение в терминах поверхностей, построенных из плакетов сильно взаимодействуюшей решетчатой КХД [11], и, до некоторой степени, инкарнация феноменологии Редже [9] в рамках КХД [12].

\footnotetext{
* Санкт-Петербургский государственный университет, Санкт-Петербург, Россия; Istituto Nazionale di Fisica Nuclear, Bologna, Italy. E-mail: andrianov@bo.infn.it

${ }^{\dagger}$ Universitat de Barcelona, Barcelona, Spain. E-mail: espriu@ecm.ub.es
} 
До сих пор не ясно, что является феноменологически приемлемым КХД-струнным действием, хотя имеются основания считать, что в определенном кинематическом режиме действия струны Намбу-Гото или Полякова приводят к удовлетворительным результатам. В данной работе мы в основном уделяем внимание низкоэнергетическим свойствам порожденных струной частичных состояний; давно известно, что выведенные из струн такого типа адронные амплитуды с неизбежностью оказываются неправильными по нескольким причинам. В качестве наиболее впечатляющего примера приведем исходную венециановскую амплитуду [1], которая выводится из струны Намбу-Гото. Она предположительно описывает рассеяние амплитуд с четырьмя пионами

$$
\mathcal{A}(\pi \pi \rightarrow \pi \pi) \sim \frac{\Gamma(-\alpha(s)) \Gamma(-\alpha(t))}{\Gamma(-\alpha(s)-\alpha(t))}+\text { перестановки по каналам, }
$$

где $\alpha(s)=1+\alpha^{\prime} s-$ (линейная) траектория Редже, характеризующая спин частицы как функцию ее массы. Наклон траектории Редже подбирается так, чтобы быть равным $\alpha^{\prime} \simeq 0.9 \Gamma_{\ni} \mathrm{B}^{-2}[13]$. Вследствие свойств Г-функций в $s$-канале имеются полюсы при $\alpha^{\prime} s=n-1$, а потому при $n=0$ возникает тахион. Более того, второе $(J=1)$ состояние (которое следует отождествить с $\rho$-частицей) является безмассовым. Наконец, такая амплитуда не обладает надлежашим адлеровым нулем, т.е. тем свойством, что при $s=t=0$ амплитуда пионного рассеяния обрашается в нуль. Мы отсылаем читателя к работам [9], [14], где более подробно обсуждаются различные попытки разрешения перечисленных выше задач посредством модификации амплитуды Венециано, и переходим к основанному на КХД решению, которое будет рассматриваться в данной статье.

Представляется вполне правдоподобным, что основная причина присутствия тахиона в спектре и неправильных киральных свойств состоит в неверном выборе вакуума [15]. Можно провести параллель с теорией скалярного поля с потенциалом $V(\phi)=-\mu^{2} \phi^{2}+$ $\lambda \phi^{4}$, порождающим спонтанное нарушение симметрии с разумным основным состоянием, но при этом возмушение вокруг $\phi=0$ дает отрицательные значения $m^{2}$ для всех компонент. Таким образом, мы предполагаем, что струнные амплитуды, получаемые посредством использования канонических вертексных операторов, соответствуют амплитудам для возбуждений вокруг неправильного, нефизического вакуума.

Возможный способ учета непертурбативных свойств КХД-вакуума был предложен в работе [16] и развит в статье [14]. А именно, можно полагать, что в КХД происходит нарушение киральной симметрии, и безмассовые (в киральном пределе) псевдоскалярные мезоны образуют фоновый КХД-вакуум, тогда как другие массивные возбуждения собраны в струну. Безмассовые псевдоскаляры можно собрать в унитарную матрицу $U(x)$. Она преобразуется как $U(x) \rightarrow U^{\prime}(x)=L U(x) R^{\dagger}$ под действием киральных преобразований, принадлежаших к $S U(2)_{\mathrm{L}} \times S U(2)_{\mathrm{R}}$ (где мы ограничиваемся рассмотрением нестранных голдстоуновских мезонов), и описывает возбуждения вокруг непертурбативного вакуума. С точки зрения теории струн $U(x)$ является не чем иным, как набором констант взаимодействий, включающих струнную переменную $x_{\mu}(\tau, \sigma)$. Нашей целью является описание самосогласованного распространения струны на этом непертурбативном фоне. Унитарная матрица $U(x)$ должна каким-то образом взаимодействовать с границей струны, где и “живут” фолейворы. 
Существенным свойством теории струн является, конечно, конформная инвариантность. В пределе больших $N_{c}$ КХД-адронные амплитуды насышаются глюонными диаграммами в виде рыболовных сетей, среди которых любые графы высшего порядка дают, в принципе, сравнимый вклад. Поэтому представляется вероятным, что не должно быть зависимости от конкретного выбора конфигурации, описываюшей распространение доминирующего глюона. Следовательно, адронное струнное действие должно подчиняться (приближенной) репараметризационной инвариантности диаграммных поверхностей и конформной инвариантности. Поскольку конформная инвариантность должна выполняться при возмущении струны вокруг любого вакуума, мы требуем, чтобы новое взаимодействие с живущими на границе киральными полями сохраняло конформную инвариантность (ср. с [17]).

В настоящей работе мы вводим обшее репараметризационно-инвариантное граничное взаимодействие с киральными полями и получаем все расходимости, индуцированные этим взаимодействием. Для перенормировки расходимостей нам понадобятся дополнительные размерные операторы в граничном действии. Из условий обрашения в нуль $\beta$-функций для $U(x)$ мы получаем уравнения движения для киральных полей в рамках разложения по малым импульсам (производным). Самосогласованным образом мы реализуем условие унитарности для киральных полей и локальность кирального лагранжиана и, наконец, вычисляем $O\left(p^{4}\right)$-члены в эффективном лагранжиане Гассера и Лейтвилера [18]. Оказалось, что наши результаты удивительно хорошо согласуются с феноменологическими значениями. В заключительном разделе мы обсуждаем ограничения данной "игрушечной” модели и указываем некоторые возможности того, как сделать ее более реалистичной.

\section{2. ГРАНИЧНОЕ ПИОННОЕ ВЗАИМОДЕЙСТВИЕ С КХД-СТРУНОЙ И ДИАГРАММЫ}

Адронная струна в конформной калибровке описывается следующим действием конформной теории поля, таргет-пространством которой является четырехмерное евклидово пространство-время ${ }^{1)}$ :

$$
\mathcal{W}_{\mathrm{str}}=\frac{1}{4 \pi \alpha^{\prime}} \int d^{2+\epsilon} \sigma\left(\frac{\varphi}{\mu}\right)^{-\epsilon} \partial_{i} x_{\mu} \partial_{i} x_{\mu}
$$

где при $\epsilon=0$ мы полагаем $x_{\mu}=x_{\mu}(\tau, \sigma),-\infty<\tau<\infty, 0<\sigma<\infty ; i=\tau, \sigma ; \mu=$ $1, \ldots, 4$. Конформный множитель $\varphi(\tau, \sigma)$ вводится для восстановления конформной инвариантности в размерности $2+\epsilon$. Известно, что наклон траектории Редже (связанный с обратным натяжением струны) универсален, $\alpha^{\prime} \simeq 0.9$ Гэ $\mathrm{B}^{-2}[13]$.

Мы намереваемся задать кирально-инвариантное взаимодействие содержашей мезонные поля матрицы $U(x)$ в пространстве флейворов со струнными степенями свободы, сохраняя при этом общую ковариантность по отношению к двумерным координатам

\footnotetext{
1) Прямые указания на размерность мирового листа струны [19] $(d=2)$ и таргет-пространства $[20](D=4)$ были найдены из анализа плотностей мезонных состояний при высоких (по сравнению с хагедорно-подобным ростом в эксперименте) энергиях.
} 
и конформную инвариантность относительно локальных масштабных преобразований двумерного метрического тензора.

Поскольку струнная переменная $x$ не содержит никакой зависимости от флейвора, мы вводим две безразмерные грассмановы переменные ("кварки"), живушие на границе листа струны: $\psi_{\mathrm{L}}(\tau)$ и $\psi_{\mathrm{R}}(\tau)$. Они преобразуются по фундаментальному представлению легкой флейворной группы $(S U(2)$ в данной работе). Тогда на границе $\sigma=0$ вводится локальное эрмитово действие $S_{b}=\int d \tau L_{f}$ для описания взаимодействия с фоновыми киральными полями $U(x(\tau))=\exp \left(i \pi(x) / f_{\pi}\right)$, где нормировочный масштаб выбран равным $f_{\pi} \simeq 93 \mathrm{MэB} \mathrm{(константе} \mathrm{слабого} \mathrm{пионного} \mathrm{распада).}$

Граничный лагранжиан выбирается репараметризационно-инвариантным и в минимальной форме имеет вид

$$
\begin{aligned}
L_{f}= & \frac{1}{2} i\left(\bar{\psi}_{\mathrm{L}} U(1-z) \dot{\psi}_{\mathrm{R}}-\dot{\bar{\psi}}_{\mathrm{L}} U(1+z) \psi_{\mathrm{R}}+\right. \\
& \left.+\bar{\psi}_{\mathrm{R}} U^{+}\left(1+z^{*}\right) \dot{\psi}_{\mathrm{L}}-\dot{\bar{\psi}}_{\mathrm{R}} U^{+}\left(1-z^{*}\right) \psi_{\mathrm{L}}\right) .
\end{aligned}
$$

Здесь и далее точка обозначает производную по $\tau: \dot{\psi} \equiv d \psi / d \tau$. Для получения уравнения (3) был выполнен ряд переопределений полей.

Дальнейшее ограничение следует из требования $C P$-инвариантности:

$$
U \leftrightarrow U^{+}, \quad \psi_{\mathrm{L}} \leftrightarrow \psi_{\mathrm{R}}
$$

Указанный выше лагранжиан является $C P$-симметричным при $z=-z^{*}=i a, z=0$. Выполнение этой симметрии оказывается кардинально важным для сохранения конформной симметрии в присутствии добавляемого граничного взаимодействия.

Легко видеть, что приведенное выше действие инвариантно относительно обших координатных преобразований двумерного мирового листа. Фермионное действие автоматически является конформно-инвариантным, оно не содержит метрического тензора на двумерном мировом листе, коль скоро его можно записать в виде линейного интеграла.

Разложим функцию $U(x)$ по степеням поля струнных координат $x_{\mu}(\tau)=x_{0 \mu}+\tilde{x}_{\mu}(\tau)$ вблизи постоянной $x_{0}$ :

$$
U(x)=U\left(x_{0}\right)+\tilde{x}_{\mu}(\tau) \partial_{\mu} U\left(x_{0}\right)+\frac{1}{2} \tilde{x}_{\mu}(\tau) \tilde{x}_{\nu}(\tau) \partial_{\mu} \partial_{\nu} U\left(x_{0}\right)+\cdots \equiv U\left(x_{0}\right)+\mathcal{V}(\tilde{x}),
$$

и будем искать потенциально расходящиеся одночастично-неприводимые диаграммы. Мы классифицируем их согласно числу петель. Каждая дополнительная петля привносит степень $\alpha^{\prime}$. Здесь можно обнаружить сходство с хорошо известным разложением по производным в киральной теории возмушений [18].

Свободный фермионный пропагатор имеет вид

$$
\left\langle\psi_{\mathrm{R}}(\tau) \bar{\psi}_{\mathrm{L}}\left(\tau^{\prime}\right)\right\rangle=U^{-1}\left(x_{0}\right) \theta\left(\tau-\tau^{\prime}\right) .
$$

Если мы налагаем требование $C P$-симметрии, то для унитарного кирального поля $U(x)$ выполняется соотношение

$$
\left\langle\psi_{\mathrm{L}}(\tau) \bar{\psi}_{\mathrm{R}}\left(\tau^{\prime}\right)\right\rangle=\left\langle\psi_{\mathrm{R}}(\tau) \bar{\psi}_{\mathrm{L}}\left(\tau^{\prime}\right)\right\rangle^{\dagger}=U\left(x_{0}\right) \theta\left(\tau-\tau^{\prime}\right) .
$$


Свободный бозонньй пропагатор, спроектированный на границу, имеет вид

$$
\left\langle x_{\mu}(\tau) x_{\nu}\left(\tau^{\prime}\right)\right\rangle=\delta_{\mu \nu} \Delta\left(\tau-\tau^{\prime}\right), \quad \Delta\left(\tau \rightarrow \tau^{\prime}\right)=\Delta(0) \sim \frac{\alpha^{\prime}}{\epsilon}, \quad \partial_{\tau} \Delta\left(\tau \rightarrow \tau^{\prime}\right)=0,
$$

где последнее равенство выполнено в размерной регуляризации (см. ниже).

Нормировку струнного пропагатора можно извлечь из определения ядра $N$-точечной тахионной амплитуды открытой струны [7]

$$
\Delta\left(\tau_{j}-\tau_{l}\right)=-2 \alpha^{\prime} \ln \left(\left|\tau_{j}-\tau_{l}\right| \mu\right) .
$$

Имея в виду это определение, найдем струнный пропагатор в размерной регуляризации при ограничении на границу. Сначала вычислим импульсный интеграл в размерности $2+\epsilon$ :

$$
\Delta_{\epsilon}(\tau)=\alpha^{\prime} \Gamma\left(\frac{\epsilon}{2}\right)\left|\frac{\tau \mu \sqrt{\pi}}{\varphi}\right|^{-\epsilon} .
$$

Для того чтобы воспроизвести выражение (9), этот размерно регуляризованньй пропагатор следует должным образом нормировать. Это можно сделать, вычитая из (10) его же значение при $\tau \mu=1$ :

$$
\left.\Delta_{\epsilon}(\tau)\right|_{\mathrm{reg}}=\alpha^{\prime} \Gamma\left(\frac{\epsilon}{2}\right)\left\{\left|\frac{\tau \mu \sqrt{\pi}}{\varphi}\right|^{-\epsilon}-\left|\frac{\sqrt{\pi}}{\varphi}\right|^{-\epsilon}\right\} .
$$

Отсюда однозначно следует соотношение

$$
\Delta(0)=-\alpha^{\prime} \Gamma\left(\frac{\epsilon}{2}\right)\left|\frac{\sqrt{\pi}}{\varphi}\right|^{-\epsilon} \stackrel{\epsilon \rightarrow 0}{=}-2 \alpha^{\prime}\left[\frac{1}{\epsilon}+C+\ln \varphi\right]+\mathcal{O}(\epsilon) \equiv \Delta_{\epsilon}-2 \alpha^{\prime} \ln \varphi,
$$

где, следуя предписанию размерной регуляризации, мы взяли $\epsilon<0$, а потому первое слагаемое в (11) обрашается в нуль при $\tau=0$.

Двухфермионные $N$-бозонные вертексные операторы порождаются разложением (5) из производяшего функционала $Z_{b}=\left\langle e^{i S_{b}}\right\rangle$ и уравнения (3). В частности, при предельном переходе $\mathrm{L} \rightarrow \mathrm{R}$ имеем

$$
V=-\frac{1}{2}\left((1-z) \mathcal{V}(\tilde{x}) \partial_{\tau}+(1+z) \partial_{\tau}[\mathcal{V}(\tilde{x}) \ldots]\right),
$$

а при переходе $\mathrm{R} \rightarrow \mathrm{L}$ появляется эрмитово сопряженная вершина $V^{+}$.

Для реализации процесса перенормировки мы выполняем петлевое разложение (эквивалентное разложению по производным) и переходим к определению контрчленов, необходимых для того, чтобы сделать теорию конечной. Это дает бета-функционал для взаимодействия $U(x)$, обрашение которого в нуль вплоть до двухпетлевого уровня требуется для реализации условия обрашения в нуль конформной аномалии. Тот факт, что мы работаем с граничной теорией поля, делает требуемые вычисления вполне выполнимыми. По поводу подробного вывода различных фейнмановских диаграмм мы отсылаем читателя к [14], а в данной работе приведем только окончательные выражения. 


\section{3. ПЕРЕНОРМИРОВКА В ОДНОЙ ПЕТЛЕ}

Используя набор правил Фейнмана, описанных в предыдущем разделе, получаем следующий результат для расходящейся части пропагатора:

$$
\theta(A-B) \frac{1}{2} \Delta(0) U^{-1}\left\{-\partial_{\mu}^{2} U+\frac{3+z^{2}}{2} \partial_{\mu} U U^{-1} \partial_{\mu} U\right\} U^{-1}
$$

Эта расходимость исключается введением подходящего контрчлена $U \rightarrow U+\delta U$ :

$$
\delta U=\Delta(0)\left[\frac{1}{2} \partial_{\mu}^{2} U-\frac{3+z^{2}}{4} \partial_{\mu} U U^{-1} \partial_{\mu} U\right]=0
$$

Конформная симметрия восстанавливается (бета-функция равна нулю), если приведенньй выше вклад обрашается в нуль.

Найдем, для каких значений $z$ эта вариация $U$ является совместной с ее унитарностью:

$$
\delta\left(U U^{+}\right)=U \cdot \delta U^{+}+\delta U \cdot U^{+}=0
$$

Простое вычисление показывает, что это имеет место при $z= \pm i$. При других значениях $z$ из уравнения (15) следует уравнение $\partial_{\mu} U U^{-1} \partial_{\mu} U=0$, которое имеет лишь тривиальное постоянное решение в евклидовом пространстве-времени. Этот выбор отличается от сделанного в работе [16].

Соответствующее локальное классическое действие, уравнением движения для которого является (15), имеет вид

$$
W^{(2)}=\frac{f_{\pi}^{2}}{8} \int d^{4} x \operatorname{tr}\left[\partial_{\mu} U \partial_{\mu} U^{-1}+\partial_{\mu} U^{+} \partial_{\mu}\left(U^{+}\right)^{-1}\right]
$$

Этот лагранжиан является, конечно, не чем иным, как хорошо известной нелинейной сигма-моделью, широко используемой при описании пионных взаимодействий.

Таким образом, мы нашли киральное действие, индуцированное КХД-струной. Оно имеет все требуемые свойства локальности, киральной симметрии и правильного поведения при малых импульсах (адлеров нуль). Более того, оно описывает безмассовые пионы. Однако, исходя из этих аргументов, нельзя предсказать обший нормировочный масштаб $f_{\pi}$, и не удается дать неуниверсальные предсказания в этом порядке кирального разложения. Рассмотрим теперь эффективный $O\left(p^{4}\right)$-лагранжиан с тем, чтобы в конце конщов получить результаты, позволяющие дать предсказания.

Преж де чем перейти к полному двухпетлевому вычислению, нам надо проверить, является ли минимальный лагранжиан (3) достаточным для перенормировки также и вершин, содержаших бозонные коншы. Оказывается, что его недостаточно.

Найдем расходимости для вершин с внешними бозонными линиями. Введем внешнее фоновое бозонное поле $\bar{x}_{\mu}$ и разобьем $x_{\mu}=\bar{x}_{\mu}+\eta_{\mu}$. Свободный пропагатор для флуктуируюшего поля $\eta_{\mu}$ совпадает с таковым для $x_{\mu}$. 
Тогда полная расходимость в вершине с двумя фермионными и одной бозонной линией имеет вид

$$
\begin{aligned}
\theta(A-B) & \frac{1}{4} \Delta(0) U^{-1}\left\{\overline { x } _ { \mu } ( A ) ( 1 + z ) \left[-\partial_{\mu}\left(\partial^{2} U\right)+2 \partial_{\nu} U U^{-1} \partial_{\mu} \partial_{\nu} U+\right.\right. \\
& \left.+(1+z) \partial_{\mu} \partial_{\nu} U U^{-1} \partial_{\nu} U-\frac{1}{2}(1+z)(3-z) \partial_{\nu} U U^{-1} \partial_{\mu} U U^{-1} \partial_{\nu} U\right]+ \\
& +\bar{x}_{\mu}(B)(1-z)\left[-\partial_{\mu}\left(\partial^{2} U\right)+(1-z) \partial_{\nu} U U^{-1} \partial_{\mu} \partial_{\nu} U+2 \partial_{\mu} \partial_{\nu} U U^{-1} \partial_{\nu} U-\right. \\
& \left.\left.-\frac{1}{2}(1-z)(3+z) \partial_{\nu} U U^{-1} \partial_{\mu} U U^{-1} \partial_{\nu} U\right]\right\} U^{-1} \equiv \\
\equiv & -\frac{1}{2} \theta(A-B) U^{-1}\left[\bar{x}_{\mu}(B) \Phi_{\mu}^{(1)}+\bar{x}_{\mu}(A) \Phi_{\mu}^{(2)}\right] U^{-1}
\end{aligned}
$$

Ампутируем теперь фермионные коншы и вычислим чисто вершинные расходимости, соответствуюшие следующему оператору в лагранжиане:

$$
\frac{i}{2}\left(\bar{\psi}_{\mathrm{L}} \Phi^{(1)} \dot{\psi}_{\mathrm{R}}-\dot{\bar{\psi}}_{\mathrm{L}} \Phi^{(2)} \psi_{\mathrm{R}}\right)+\text { э.c. }, \quad \Phi^{(1,2)} \equiv \bar{x}_{\mu}(\tau) \Phi_{\mu}^{(1,2)}
$$

где вершинную матрицу $\Phi_{\mu}^{(1,2)}$ можно реорганизовать следующим образом:

$$
\begin{aligned}
\Phi_{\mu}^{(1)}= & \Delta(0)\left\{(1-z) \partial_{\mu}\left(\frac{1}{2} \partial^{2} U-\frac{3+z^{2}}{4} \partial_{\nu} U U^{-1} \partial_{\nu} U\right)-\right. \\
& -\frac{1-z^{2}}{2}\left(\frac{1-z}{2} \partial_{\mu} \partial_{\nu} U U^{-1} \partial_{\nu} U-\frac{1+z}{2} \partial_{\nu} U U^{-1} \partial_{\mu} \partial_{\nu} U+\right. \\
& \left.\left.+z \partial_{\nu} U U^{-1} \partial_{\mu} U U^{-1} \partial_{\nu} U\right)\right\} \equiv(1-z) \partial_{\mu}(\delta U)-\phi_{\mu}, \\
\Phi_{\mu}^{(2)}= & (1+z) \partial_{\mu}(\delta U)+\phi_{\mu} .
\end{aligned}
$$

Члены, пропорциональные производным $\delta U$, автоматически исключаются перенормировкой однопетлевого пропагатора (и обращаются в нуль, если предполагается вьполнение уравнений движения). Однако часть, пропорциональная $\phi_{\mu}$, остается, и для поглошения этих расходимостей требуются новые контрчлены, которые можно параметризовать тремя затравочными константами $g_{1}, g_{2}$ и $g_{3}$, являюшимися вешественными, если имеет место $C P$-симметрия при $z=-z^{*}$ :

$$
\begin{aligned}
\Delta L_{\mathrm{bare}}= & \frac{i}{8}\left(1-z^{2}\right) \bar{\psi}_{\mathrm{L}}\left(\left(g_{1}-z g_{2}\right) \partial_{\nu} \dot{U} U^{-1} \partial_{\nu} U-\left(g_{1}+z g_{2}\right) \partial_{\nu} U U^{-1} \partial_{\nu} \dot{U}+\right. \\
& \left.+2 z g_{3} \partial_{\nu} U U^{-1} \dot{U} U^{-1} \partial_{\nu} U\right) \psi_{\mathrm{R}}+\text { э.c. }
\end{aligned}
$$

Перенормировка выполняется переопределением спариваний $g_{i}$ следуюшим образом:

$$
g_{i}=g_{i, r}-\Delta(0)
$$


Постоянные $g_{i, r}$ являются конечными, но в принципе зависящими от схемы, и из (12) следует, что логарифмическая зависимость затравочных спариваний от конформного множителя $\varphi$ вводится в процессе перенормировки. Контрчлены имеют более высокую размерность, чем исходный лагранжиан $(3)$, и поэтому спаривания $g_{i}$ имеют размерность $M^{-2}$. Поскольку (3) дает наиболее обшее спаривание, допускаемое симметриями модели, мы приходим к выводу, что конформная симметрия представляется нарушенной этими граничными спариваниями уже на древесном уровне, если только не произойдет их обрашения в нуль.

Однако несмотря на то, что новые спаривания являются размерными, оказывается, что их вклад в след тензора энергии-импульса обрашается в нуль, если учесть требования унитарности $U$ (см. следующий раздел), а потому конформная инвариантность не нарушена в том порядке, в котором мы работаем.

Во всяком случае, появление новых вершин изменяет фермионный пропагатор. Из таких членов (которые имеют высший порядок по производным) получаем следующий вклад в пропагатор:

$$
\begin{aligned}
& \theta(A-B) \frac{1}{16} \Delta(0)\left(1-z^{2}\right) U^{-1}\left\{2\left(g_{1, r}-z^{2} g_{2, r}\right) \partial_{\rho} U U^{-1} \partial_{\mu} \partial_{\rho} U U^{-1} \partial_{\mu} U-\right. \\
&-(1+z)\left(g_{1, r}+z g_{2, r}\right) \partial_{\rho} U U^{-1} \partial_{\mu} U U^{-1} \partial_{\rho} \partial_{\mu} U- \\
&-(1-z)\left(g_{1, r}-z g_{2, r}\right) \partial_{\rho} \partial_{\mu} U U^{-1} \partial_{\rho} U U^{-1} \partial_{\mu} U+ \\
&\left.+4 z^{2} g_{3, r} \partial_{\rho} U U^{-1} \partial_{\mu} U U^{-1} \partial_{\rho} U U^{-1} \partial_{\mu} U\right\} U^{-1} \equiv \\
& \equiv-\theta(A-B) \Delta(0) U^{-1} \delta^{(4)} U U^{-1} .
\end{aligned}
$$

Мы обозначим этот вклад как $\theta(A-B) d_{g}$. К однопетлевому результату следует добавить расходимость, содержашуюся в $d_{g}$, тем самым модифицируя перенормировку и уравнения движения поля $U$ :

$$
\bar{\delta} U=\Delta(0)\left[\frac{1}{2} \partial_{\mu}^{2} U-\frac{3+z^{2}}{4} \partial_{\mu} U U^{-1} \partial_{\mu} U+\delta^{(4)} U\right]=0
$$

Это является одним из источников $O\left(p^{4}\right)$-членов. Однако, если бы это было единственной модифицикацией, мы столкнулись бы с серьезной проблемой, поскольку новые добавки к уравнениям движения не могут быть получены ни из какого локального лагранжиана, и, кроме того, не сохраняют пертурбативную унитарность $U$. K счастью, как мы увидим, имеется и другой вклад.

Что касается других вершин в (13), можно доказать [14], что любая диаграмма с произвольным числом внешних бозонных линий и двумя фермионными линиями, т.е. любая вершина, порожденная пертурбативным разложением (3), делается конечной с помошью указанных выше контрчленов. Этим завершается программа переномировки в одной петле.

\section{4. ОБРАШЕНИЕ В НУЛЬ КОНФОРМНОЙ АНОМАЛИИ}

Обратная связь между граничным взаимодействием и струнным сектором обеспечивается фермионным детерминантом. В лагранжиане (3) имеются два взаимно сопря- 
женных оператора в билинейной форме:

$$
\begin{aligned}
\mathcal{D} & =\frac{i}{2}\left[(1-z) U(\tau) i \partial_{\tau}+(1+z) i \partial_{\tau}(U(\tau))\right], \\
\mathcal{D}^{\dagger} & =\frac{i}{2}\left[\left(1+z^{*}\right) U^{+}(\tau) i \partial_{\tau}+\left(1-z^{*}\right) i \partial_{\tau}\left(U^{+}(\tau)\right)\right] .
\end{aligned}
$$

Поэтому полньй фермионный детерминант (результат интегрирования по фермионам), или фермионный петлевой вклад, можно представить в виде

$$
Z_{f}=\left\|\mathcal{D D}^{\dagger}\right\|=\left\|\left(i \partial_{\tau}-\frac{i}{2}(1-z) \dot{U} U^{+}\right)\left(i \partial_{\tau}-\frac{i}{2}\left(1-z^{*}\right) \dot{U} U^{+}\right)\right\|,
$$

где мы ограничились унитарными полями $U$. Теперь можно факторизовать бесконечную постоянную, связанную со свободными операторами, и найти нетривиальную часть в терминах фермионых пропагаторов:

$$
\begin{aligned}
Z_{f}= & \left\|\left(i \partial_{\tau}\right)^{2}\right\| \exp \left\{\operatorname { t r } \left(\ln \left(1-\frac{i}{2 i \partial_{\tau}}(1-z) \dot{U} U^{+}\right)+\right.\right. \\
& \left.\left.+\ln \left(1-\frac{i}{2 i \partial_{\tau}}\left(1-z^{*}\right) \dot{U} U^{+}\right)\right)\right\}= \\
= & C \exp \left\{-\theta(0) \frac{1}{2} \int_{-\infty}^{\infty} d \tau\left(1-z+1-z^{*}\right) \operatorname{tr}\left(\dot{U} U^{+}\right)\right\}= \\
= & C \exp \left\{-\frac{1}{2} \int_{-\infty}^{\infty} d \tau \operatorname{tr}\left(\dot{U} U^{+}\right)\right\} .
\end{aligned}
$$

Здесь было использовано треугольное свойство свободного фермионного пропагатора

$$
\left\langle\tau\left|\frac{i}{i \partial_{\tau}+i \epsilon}\right| \tau^{\prime}\right\rangle=\theta\left(\tau-\tau^{\prime}\right)
$$

которое следует из предписания, состоящего в добавлении $+i \epsilon$ в опережающих функциях Грина. В результате при взятии функционального следа остается только первый порядок в разложении логарифмов в (27). Здесь мы использовали значение $\theta(0)=1 / 2$ и $C P$-инвариантый выбор $z=-z^{*}$.

Очевидно, что для групп $S U(N)$ и $U(1)$ с периодическими граничными условиями экспонента в (27) обрашается в нуль, и поэтому фермионный петлевой вклад от минимального лагранжиана отсутствует.

Вывод фермионного детерминанта для расширенного лагранжиана, включающего контрчлены высших размерностей (21), аналогичен. Соответствуюшие дифференциальные операторы выглядят сходным образом, но имеют расширенные потенциальные члены. Как и прежде, только первый порядок в разложении логарифмического следа не обращается в нуль:

$$
\begin{aligned}
\ln Z_{f}= & C^{\prime}+\frac{1}{8} \int_{-\infty}^{\infty} d \tau \operatorname{tr}\left(\left(1-z^{2}\right)\left[z g_{2} \partial_{\nu} \dot{U} \partial_{\nu} U^{+}+z g_{3} \partial_{\nu} U U^{+} \dot{U} U^{+} \partial_{\nu} U U^{+}\right]+\right. \\
& \left.+\left(1-\left(z^{*}\right)^{2}\right)\left[-z^{*} g_{2} \partial_{\nu} \dot{U} \partial_{\nu} U^{+}+z^{*} g_{3} \partial_{\nu} U U^{+} \dot{U} U^{+} \partial_{\nu} U U^{+}\right]\right) .
\end{aligned}
$$


Операторы, пропорциональные $g_{1}$, не появляются после взятия следа. Выберем $C P$ симметричные постоянные $z=-z^{*}$. Оказывается, что операторы, содержашие константу взаимодействия $g_{3}$, пропорциональны $z+z^{*}$ ипотому обращаются в нуль. Что же касается $g_{2}$-членов, то они образуют полную производную по времени в $C P$-инвариантном случае:

$$
\ln Z_{f}=C^{\prime}+\frac{1}{8} \int_{-\infty}^{\infty} d \tau\left(1-z^{2}\right) z g_{2} \partial_{\tau} \operatorname{tr}\left(\partial_{\nu} U \partial_{\nu} U^{+}\right)=0
$$

причем последнее имеет место для периодических граничных условий.

Таким образом, несмотря на то что вершины высших размерностей привносят в лагранжиан зависимость от масштаба (постоянные $g_{j}$ являются размерными), они не порождают масштабной аномалии из-за фермионных петель, если и только если на лагранжиан наложено условие $C P$-симметрии.

\section{5. ПЕРЕНОРМИРОВКА ФЕРМИОННОГО ПРОПАГАТОРА В ДВУХ ПЕТЛЯХ}

Имеются 10 двухпетлевых одночастично-неприводимых диаграмм, которые перечислены и аналитически вычислены в [14]. Расходимости в пропагаторе в двух петлях можно разделить на пять частей:

$$
\theta(A-B)\left[d_{\mathrm{I}}+d_{\mathrm{II}}+d_{\mathrm{III}}+d_{\mathrm{IV}}+d_{\mathrm{V}}\right]
$$

Первая и вторая части содержат двойные расходимости $\Delta^{2}(0)$, третья, четвертая и пятая - единственную расходимость $\Delta(0)$.

Компонента $d_{\mathrm{I}}$ представляет собой "вторую вариацию", или однопетлевую расходимость в однопетлевой расходимости:

$$
\begin{aligned}
d_{\mathrm{I}}= & -\frac{1}{2} U^{-1} \delta(\delta U) U^{-1}=-\frac{1}{2} \Delta(0) U^{-1}\left\{\frac{1}{2} \partial_{\mu}^{2}(\delta U)-\frac{3+z^{2}}{4}\left[\partial_{\mu}(\delta U) U^{-1} \partial_{\mu} U-\right.\right. \\
& \left.\left.-\partial_{\mu} U U^{-1} \delta U U^{-1} \partial_{\mu} U+\partial_{\mu} U U^{-1} \partial_{\mu}(\delta U)\right]\right\} U^{-1}, \\
\delta U= & \Delta(0)\left[\frac{1}{2} \partial_{\mu}^{2} U-\frac{3+z^{2}}{4} \partial_{\mu} U U^{-1} \partial_{\mu} U\right] .
\end{aligned}
$$

Поэтому она перенормируется переопределением поля $U$ и обрашается в нуль, когда накладываются уравнения движения. Контрчлены, перенормирующие поле $U$, приводят к тому же выражению, но в два раза большему и имеюшему противоположный знак в соответствии с аргументами, основанными на ренормализационной группе.

Вторая часть представляет собой остающееся в двухпетлевых диаграммах после вычитания $d_{\mathrm{I}}$ слагаемое порядка $\Delta^{2}(0)$ и имеет вид

$$
\begin{aligned}
d_{\mathrm{II}}= & \frac{1}{32} \Delta^{2}(0)\left(1-z^{2}\right) U^{-1}\left\{2\left(1-z^{2}\right) \partial_{\rho} U U^{-1} \partial_{\mu} \partial_{\rho} U U^{-1} \partial_{\mu} U-\right. \\
& -(1+z)^{2} \partial_{\rho} U U^{-1} \partial_{\mu} U U^{-1} \partial_{\rho} \partial_{\mu} U-(1-z)^{2} \partial_{\rho} \partial_{\mu} U U^{-1} \partial_{\rho} U U^{-1} \partial_{\mu} U+ \\
& \left.+4 z^{2} \partial_{\rho} U U^{-1} \partial_{\mu} U U^{-1} \partial_{\rho} U U^{-1} \partial_{\mu} U\right\} U^{-1} .
\end{aligned}
$$


Это слагаемое тождественно (но имеет противоположный знак) вкладу, порожденному однопетлевым контрчленом в вершине с двумя фермионами и одной бозонной линией после подстановки вершины в однопетлевую диаграмму.

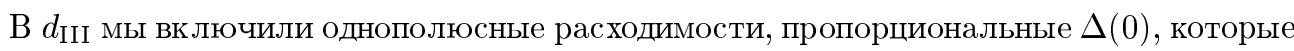
исчезают, как только учитывается однопетлевая перенормировка $U$ в конечной нелокальной части фермионного пропагатора в одной петле, т.е. при замене $U$ на $U+\delta^{(2)} U$ в однопетлевом пропагаторе:

$$
\begin{aligned}
& \frac{1}{4}\left(1-z^{2}\right) \Delta(0) \Delta(A, B) U^{-1}\left[\partial_{\mu}(\delta U) U^{-1} \partial_{\mu} U-\right. \\
& \left.\quad-\partial_{\mu} U U^{-1} \delta U U^{-1} \partial_{\mu} U+\partial_{\mu} U U^{-1} \partial_{\mu}(\delta U)\right] U^{-1}
\end{aligned}
$$

Аналогичным образом в $d_{\mathrm{IV}}$ мы включили расходимости, которые устраняются, когда в конечную часть однопетлевого фермионного пропагатора включаются дополнительные контрчлены в однобозонных вершинах (пропорциональных $g_{i}$ ). Можно проверить [14], что все линейные по $\Delta(0)$ и по $\Delta(A, B)$ слагаемыев двухпетлевом фермионном пропагаторе принадлежат или к $d_{\mathrm{III}}$, или к $d_{\mathrm{IV}}$.

Однако остаются некоторые однополюсные расходимости. Действительно, имеются некоторые расходимости, линейные по $\Delta(0)$, которые получаются из двойного интеграла в неприводимых двухпетлевых диаграммах с максимальным числом вершин:

$$
\begin{aligned}
J(A, B) & =\int_{B}^{A} d \tau_{1} \int_{B}^{\tau_{1}} d \tau_{2} \partial_{\tau_{1}} \Delta\left(\tau_{1}-\tau_{2}\right) \partial_{\tau_{2}} \Delta\left(\tau_{1}-\tau_{2}\right)= \\
& =-\int_{0}^{A-B} d \tau(A-B-\tau)[\dot{\Delta}(\tau)]^{2} .
\end{aligned}
$$

Этот интеграл был вычислен с использованием двух различных регуляризаций. Расходимость получается равной

$$
\begin{aligned}
d_{\mathrm{V}}= & c_{\mathrm{V}} \Delta(0)\left[U^{-1} \partial_{\rho} U U^{-1} \partial_{\mu} U U^{-1} \partial_{\mu} U U^{-1} \partial_{\rho} U U^{-1}-\right. \\
& \left.-U^{-1} \partial_{\rho} U U^{-1} \partial_{\mu} U U^{-1} \partial_{\rho} U U^{-1} \partial_{\mu} U U^{-1}\right] \equiv \\
\equiv & -\Delta(0) U^{-1} \bar{d}_{\mathrm{V}} U^{-1}
\end{aligned}
$$

где $c_{\mathrm{V}}=\alpha^{\prime}\left(1-z^{2}\right)^{2} / 8=\alpha^{\prime} / 2$ при $z= \pm i$. Это слагаемое, остающееся после добавления всех контрчленов, а также (23) являются единственными истинными новыми расходимостями, которые могут давать вклад в бета-функцию (одинарные полюсы). Поэтому его необходимо добавить в уравнения движения в следуюшем порядке $\alpha^{\prime}$-разложения, и оно сушественным образом модифиширует член $\delta^{(4)} U$ :

$$
\delta^{(4)} U \rightarrow \delta^{(4)} U+\bar{d}_{\mathrm{V}} .
$$

Действительно, новые расходимости допускают ненулевые решения для констант взаимодействия $g_{i}$ и, следовательно, ненулевые значения $O\left(p^{4}\right)$ коэффициентов ГассераЛейтвилера. 


\section{6. ЛОКАЛЬНАЯ ИНТЕГРИРУЕМОСТЬ ЧЕТЫРЕХМЕРНЫХ УРАВНЕНИЙ ДВИЖЕНИЯ}

$O\left(p^{2}\right)$-уравнения движения (15) можно получить из локального действия вайнбергова типа (17), включаюшего унитарную матрицу $U(x)$, только при $z= \pm i$. Если соответствуюшие слагаемые с четырьмя производными, которые мы нашли в предыдущих разделах, должны быть выведены из операторов размерности четыре в локальном эффективном лагранжиане, то следует наложить определенные связи на постоянные $g_{i, r}$.

Такой лагранжиан имеет только два слагаемых, совместных с киральной симметрией, если мы используем уравнения движения размерности два (15):

$$
\mathcal{L}^{(4)}=\frac{1}{2} f_{\pi}^{2} \operatorname{tr}\left(K_{1} \partial_{\mu} U \partial_{\rho} U^{-1} \partial_{\mu} U \partial_{\rho} U^{-1}+K_{2} \partial_{\mu} U \partial_{\mu} U^{-1} \partial_{\rho} U \partial_{\rho} U^{-1}+\text { э.c. }\right) .
$$

Члены $\partial_{\mu}^{2} U \partial_{\rho} U^{-1} \partial_{\rho} U U^{-1}, \partial_{\mu}^{2} U \partial_{\rho}^{2} U^{-1},\left(\partial_{\mu}^{2}\right)^{2} U U^{-1}, \partial_{\mu} \partial_{\rho} U \partial_{\mu} \partial_{\rho} U^{-1}$, которые в принципе могут возникать, редуцируются к набору (38) с помощью интегрирования по частям в действии и с помошью уравнений движения размерности два (15).

Вариация приведенного выше лагранжиана дает следуюшую добавку к уравнениям движения:

$$
\begin{aligned}
\frac{\delta S}{\delta U}= & -f_{\pi}^{2} U^{-1}\left\{2 K _ { 1 } \left[\partial_{\mu} \partial_{\rho} U U^{-1} \partial_{\mu} U U^{-1} \partial_{\rho} U+\partial_{\mu} U U^{-1} \partial_{\rho} U U^{-1} \partial_{\mu} \partial_{\rho} U-\right.\right. \\
& -\partial_{\mu} U U^{-1} \partial_{\rho} U U^{-1} \partial_{\mu} U U^{-1} \partial_{\rho} U-2 \partial_{\mu} U U^{-1} \partial_{\rho} U U^{-1} \partial_{\rho} U U^{-1} \partial_{\mu} U+ \\
& \left.+\partial_{\mu} U U^{-1} \partial_{\rho}^{2} U U^{-1} \partial_{\mu} U\right]+ \\
& +K_{2}\left[\partial_{\mu} \partial_{\rho} U U^{-1} \partial_{\mu} U U^{-1} \partial_{\rho} U+\partial_{\mu} U U^{-1} \partial_{\rho} U U^{-1} \partial_{\mu} \partial_{\rho} U+\right. \\
& +2 \partial_{\mu} U U^{-1} \partial_{\mu} \partial_{\rho} U U^{-1} \partial_{\rho}+\partial_{\mu}^{2} U U^{-1} \partial_{\rho} U U^{-1} \partial_{\rho} U+\partial_{\mu} U U^{-1} \partial_{\mu} U^{-1} \partial_{\rho}^{2} U- \\
& -2 \partial_{\mu} U U^{-1} \partial_{\rho} U U^{-1} \partial_{\mu} U U^{-1} \partial_{\rho} U-\partial_{\mu} U U^{-1} \partial_{\rho} U U^{-1} \partial_{\rho} U U^{-1} \partial_{\mu} U- \\
& \left.\left.-3 \partial_{\mu} U U^{-1} \partial_{\mu} U U^{-1} \partial_{\rho} U U^{-1} \partial_{\rho} U\right]\right\} U^{-1}
\end{aligned}
$$

Отождествим эту параметризацию констант с константами взаимодействия, возникающими из уравнений движения (24), дополненных соотношениями (37) после того, как $O\left(p^{2}\right)$-уравнения движения применены для устранения лапласиана на киральных полях $\partial_{\mu}^{2} U$. Тогда получается следуюший набор коэффициентов при различных структурах кирального поля:

$$
\begin{aligned}
-2\left(2 K_{1}+K_{2}\right) & =\frac{1}{16}\left(1-z^{2}\right)(1+z)\left(g_{1, r}+z g_{2, r}\right)=\frac{1}{16}\left(1-z^{2}\right)(1-z)\left(g_{1, r}-z g_{2, r}\right) ; \\
-4 K_{2} & =\frac{1}{8}\left(1-z^{2}\right)\left(-g_{1, r}+z^{2} g_{2, r}\right) ; \quad 2\left[\left(1-z^{2}\right) K_{1}+K_{2}\right]=-c_{\mathrm{V}} ; \\
-2 z^{2} K_{2} & =0 ; \quad 4\left[K_{1}+K_{2}\right]=-\frac{1}{4}\left(1-z^{2}\right) z^{2} g_{3, r}+c_{\mathrm{V}} .
\end{aligned}
$$

При $z^{2}=-1$ возможно только одно решение:

$$
K_{2}=0, \quad K_{1}=-\frac{1}{4} c_{\mathrm{V}}=-\frac{\alpha^{\prime}}{8} ; \quad g_{1, r}=-g_{2, r}=-g_{3, r}=4 c_{\mathrm{V}} .
$$


Таким образом, сравнивая уравнения (38) с обычной параметризацией лагранжиана Гассера-Лейтвилера [18], получаем

$$
L_{1}=\frac{1}{2} L_{2}=-\frac{1}{4} L_{3}=-\frac{1}{2} K_{1} f_{\pi}^{2}=\frac{f_{\pi}^{2} \alpha^{\prime}}{16} .
$$

При $\alpha^{\prime}=0.9$ Гэ $\mathrm{B}^{-2}$ и $f_{\pi} \simeq 93 \mathrm{MэВ} \mathrm{это} \mathrm{приводит} \mathrm{к} \mathrm{значению} L_{2} \simeq 0.9 \cdot 10^{-3}$, что является вполне удовлетворительным результатом [21].

Другая проверка возникает из совместности унитарности $U$ и уравнений движения на двухпетлевом уровне. Оказывается, что если принять произвольные вешественные коэффициенты в наборе операторов размерности четыре, входящих в (39), то единственное решение, согласованное с унитарностью, будет иметь вид параметризации с постоянными $K_{1}$ и $K_{2}$. Таким образом, требование сохранения унитарности при перенормировке поля полностью эквивалентно условиям локальной интегрируемости, аналогично случаю операторов размерности два. Этот замечательный результат указывает на состоятельность всей процедуры ${ }^{2}$.

\section{7. ЗАКЛЮЧЕНИЕ И ПЕРСПЕКТИВЫ}

В данной работе мы представили упрощенную модель, или, скорее, грубую аппроксимацию КХД-струны, основанную на принципах конформной теории поля. Требование конформной инвариантности КХД-струн вблизи кирально-неинвариантного вакуума приводит к лагранжиану Гассера-Лейтвилера. В некоторый момент, однако, мы ожидаем настоящего нарушения конформной инвариантности. Это связано с конформной аномалией, которую с необходимостью должна демонстрировать теория бозонной струны при $d \neq 26$. Более того, наивное действие бозонной струны, использованное в данной работе, не препятствует сминанию больших евклидовых мировых листов [25]. Оно также не описывает корректно высокотемпературное поведение КХД при больших $N$ [26]. Для исправления этого индуцированное КХД-струнное действие может включать нелокальные операторы [5], [27] или, по крайней мере, операторы с высшими производными [28], явно нарушающие конформную симметрию на мировом листе. Эти операторы помогают сделать струны гладкими и дают правильные высокотемпературные асимптотики. Однако, поскольку мы интересуемся здесь низкоэнергетическими свойствами струн, мы не ожидаем, что подход и приемы, использованные при выводе действия кирального поля, потребуют каких-либо значительных изменений в модифицированном КХД-струнном действии. Изменения могут произойти лишь из-за поведения модифищированного струнного пропагатора на малых расстояниях.

\footnotetext{
${ }^{2)}$ Соотношение $L_{1}=(1 / 2) L_{2}=-(1 / 4) L_{3}$ было установлено ранее в моделях бозонизации [22] и в киральной кварковой модели [23] посредством разложения кваркового детерминанта по производным. Однако в то же время его возможная связь со струнным описанием КХД не была отмечена. Первая попытка вывода киральных коэффициентов из дуальных амплитуд Венециано-ЛавласаШапиро была предпринята в работе [24], где были найдены значения $L_{1}=L_{2} / 2=\left(F_{\pi}^{2} / 16 m_{\rho}^{2}\right) \ln 2$, $L_{3}=-2 L_{2}$, которые численно оказываются также вполне допустимыми. Однако выбор дуальной амплитуды в [24] не может быть связан ни с какой известной адронной струной.
} 
Наконец, отметим некоторые пробелы в подходе, предпринятом в данной работе, и очертим перспективы дальнейшего развития.

1. Мы ограничились глобальной флейвор-группой $S U(2)$. В этом случае из простого фермионного лагранжиана (3) можно вывести только члены с четной четностью в уравнениях движения. С другой стороны, $S U(3)$-обобшение должно приводить также к вкладу Весса-Зумино-Виттена (ВЗВ) с нечетной четностью в уравнениях движения. Поскольку предполагается, что все существенные мезонные степени свободы воспроизводятся адронной струной, нельзя ожидать появления ВЗВ-членов из многофермионного взаимодействия, которое может эффективно возникать только из-за редукции глюболов и гибридов с большой массой, подавляемых в пределе больших $N$. Тогда единственный способ получения членов с нечетной четностью состоит в придании одномерным фермионам истинных спинорных степеней свободы, т.е., например, в добавлении репараметризационно-инвариантной вершины

$$
\Delta L_{f}=\xi \bar{\psi} \gamma^{\mu} \dot{x}_{\mu} \psi
$$

с матрицами Дирака $\gamma^{\mu}$ и новой размерной постоянной $\xi$. Это расширение будет исследовано в отдельной статье.

2. В принципе можно добавить внешние электромагнитные поля, приводящие к замене пространственно-временны́х производных ковариантными, и тем самым вычислить также киральные постоянные $L_{9}, L_{10}$. Однако пока не ясно, как ввести массы токовых кварков без противоречия с картиной открытых струн с ультрарелятивистскими кварками на концах. Поэтому вычисление других киральных постоянных требует бо́льших усилий в рамках струнного подхода, если фермионы на границе действительно связаны с кварками.

3. Возврашаясь к возможным действиям с высшими производными [28], нелокальным [5], [27] или пятимерным действиям [29] для адронной струны, отметим, что представляет интерес исследование следов отклонений от конформной теории струн в низкоэнергетических киральных постоянных и феноменологии.

Благодарности. Работа поддержана со стороны MCyT FPA2001-3598, CIRIT 2001SGR-00065 и European Networks EURODAPHNE and EUROGRID, а также гранта РФФИ № 01-02-17152, гранта Российского министерства образования № Е00-33-208 и программы "Российские университеты: фундаментальные исследования" (грант № 992612). Эта работа представляет собой вклад авторов в выполнение проекта ИНТАС 2000-587.

\section{Список литературы}

[1] G. Veneziano. Nuovo Cimento. A. 1968. V. 57. P. 190.

[2] C. Lovelace. Phys. Lett. B. 1968. V. 28. P. 264; J. Shapiro. Phys. Rev. 1969. V. 179. P. 1345; Y. Nambu. Dual hadrodynamics. In: Proc. Int. Conf. on Symmetries and Quark Models. Ed. R. Chand. New York: Gordon and Breach, 1970. P. 269; H. B. Nielsen. An almost physical interpretation of the Veneziano model. In: Proc. 15 Int. Conf. on High En. Phys. Kiev: Naukova dumka, 1970. P. 234; L. Susskind. Nuovo Cimento. A. 1970. V. 69. P. 457.

[3] G. 't Hooft. Nucl. Phys. B. 1974. V. 72. P. 461; G. Veneziano. Nucl. Phys. B. 1976. V. 117. P. 519. 
[4] A. M. Polyakov. Phys. Lett. B. 1981. V. 103. P. 207; Nucl. Phys. B. 1986. V. 268. P. 406; Г. П. Пронько, А. В. Разумов. ТМФ. 1983. Т. 56. № 2. С. 192; T. L. Curtright, G. I. Ghandour, C. B. Thorn, C. K. Zachos. Phys. Rev. Lett. 1986. V. 57. P. 799; T. Curtright, G. Ghandour, C. K. Zachos. Phys. Rev. D. 1986. V. 34. P. 3811; H. Kleinert. Phys. Lett. B. 1986. V. 174. P. 335; Phys. Rev. Lett. 1987. V. 58. P. 1915; D. C. Lewellen. Nucl. Phys. B. 1993. V. 392. P. 137.

[5] D. Antonov, D. Ebert, Y. A. Simonov. Mod. Phys. Lett. A. 1996. V. 11. P. 1905; D. Antonov. Surv. High Energy Phys. 2000. V. 14. P. 265.

[6] L. D. Solovev. Phys. Rev. D. 1998. V. 58. P. 035005.

[7] C. Rebbi. Phys. Rep. C. 1974. V. 12. P. 1.

[8] J. Scherk. Rev. Mod. Phys. 1975. V. 47. P. 123.

[9] P. Frampton. Dual Resonance Models. New York: Benjamin, 1974.

[10] A. Neveu. Dual resonance models and strings in QCD. In: Recent Advances in Field Theory and Statistical Mechanics (Session XXXIX of the Summer School in the Theoretical Physics Held at the Les Houches, August 2 - September 10, 1982). Eds. J.-B. Zuber, R. Stora. Amsterdam: North-Holland, 1982. P. 757; A. A. Migdal. Phys. Rep. 1983. V. 102. P. 199; A. M. Polyakov. Gauge Fields and Strings. Chur, Switzerland: Harwood, 1987; М. Грин, Дж. Швари, Э. Виттен. Теория суперструн. М.: Мир, 1990; J. Polchinski. Strings and QCD. In: Black Holes, Membranes, Wormholes and Superstrings. Eds. S. Kalara, D. Nanopoulos. Singapore: World Scientific, 1993. P. 220; hep-th/9210045.

[11] K. G. Wilson. Phys. Rev. D. 1974. V. 10. P. 2445.

[12] R. Kirschner, L. N. Lipatov, L. Szymanowski. Nucl. Phys. B. 1994. V. 425. P. 579.

[13] S. Filipponi, G. Pancheri, Y. Srivastava. Phys. Rev. Lett. 1998. V. 80. P. 1838.

[14] J. Alfaro, A. A. Andrianov, L. Balart, D. Espriu. Hadronic string, conformal invariance and chiral symmetry. hep-th/0203215.

[15] E. Cremmer, J. Scherk. Nucl. Phys. B. 1974. V. 72. P. 117.

[16] J. Alfaro, A. Dobado, D. Espriu. Phys. Lett. B. 1999. V. 460. P. 447.

[17] C. G. Callan, E. J. Martinec, M. J. Perry, D. Friedan. Nucl. Phys. B. 1985. V. 262. P. 593.

[18] J. Gasser, H. Leutwyler. Nucl. Phys. B. 1985. V. 250. P. 465.

[19] S. Fubini, A. J. Hanson, R. Jackiw. Phys. Rev. D. 1973. V. 7. P. 1732; A. Strumia, G. Venturi. Lett. Nuovo Cimento. 1975. V. 13. P. 337.

[20] K. R. Dienes, J.-R. Cudell. Phys. Rev. Lett. 1994. V. 72. P. 187.

[21] G. Amoros, J. Bijnens, P. Talavera. Nucl. Phys. B. 2001. V. 602. P. 87.

[22] A. A. Andrianov, L. Bonora. Nucl. Phys. B. 1984. V. 233. P. 232; P. 247; A. A. Andrianov. Phys. Lett. B. 1985. V. 157. P. 425.

[23] D. Espriu, E. de Rafael, J. Taron. Nucl. Phys. B. 1990. V. 345. P. 22; Erratum. 1991. V. 355. P. 278.

[24] M. V. Polyakov, V. V. Vereshagin. Phys. Rev. D. 1996. V. 54. P. 1112.

[25] A. M. Polyakov. Physica Scripta T. 1987. V. 15. P. 191.

[26] J. Polchinski, Zhu Yang. Phys. Rev. D. 1992. V. 46. P. 3667.

[27] A. M. Polyakov. Nucl. Phys. B. 1997. V. 486. P. 23; F. Quevedo, C. A. Trugenberger. Nucl. Phys. B. 1997. V. 501. P. 143; D. Antonov, D. Ebert. Phys. Rev. D. 1998. V. 58. P. 067901.

[28] M. C. Diamantini, H. Kleinert, C. A. Trugenberger. Phys. Rev. Lett. 1999. V. 82. P. 267; Phys. Lett. B. 1999. V. 457. P. 87.

[29] A. M. Polyakov. Nucl. Phys. Proc. Supp. 1998. V. 68. P. 1; Int. J. Mod. Phys. A. 1999. V. 14. P. 645; E. Alvarez, C. Gomez, T. Ortin. Nucl. Phys. B. 1999. V. 545. P. 217; E. Alvarez, C. Gomez. Nucl. Phys. B. 1999. V. 550. P. 169; JHEP. 2000. V. 0005. P. 012. 